Research Article

\title{
Optimization of Storage Location Assignment in Tier-To-Tier Shuttle-Based Storage and Retrieval Systems Based on Mixed Storage
}

\author{
Bin Lei $\mathbb{D},{ }^{1,2,3}$ Fangxin Hu, ${ }^{1}$ Zhaoyuan Jiang $\mathbb{D}^{1,2}$ and Haibo Mu $\mathbb{D}^{4}$ \\ ${ }^{1}$ Mechatronics T\&R Institute, Lanzhou Jiaotong University, Lanzhou 730070, China \\ ${ }^{2}$ Engineering Technology Center for Informatization of Logistics \& Transport Equipment, Lanzhou 730070, China \\ ${ }^{3}$ Gansu Provincial Industry Technology Center of Logistics \& Transport Equipment, Lanzhou 730070, China \\ ${ }^{4}$ School of Traffic and Transportation, Lanzhou Jiaotong University, Lanzhou 730070, China
}

Correspondence should be addressed to Bin Lei; 317165496@qq.com

Received 5 December 2019; Revised 27 February 2020; Accepted 4 May 2020; Published 20 May 2020

Academic Editor: Sébastien Poncet

Copyright ( $\odot 2020$ Bin Lei et al. This is an open access article distributed under the Creative Commons Attribution License, which permits unrestricted use, distribution, and reproduction in any medium, provided the original work is properly cited.

\begin{abstract}
To improve the efficiency of tier-to-tier shuttle-based storage and retrieval system (SBS/RS), the optimization problem of the location allocation based on the mixed storage of goods is proposed. Considering the effect of warehouse operation scheduling and batch outbound allocation on the location allocation, an optimization model with the shortest outbound time of all outbound orders in a certain historical period is established. The optimization model consists of two stages: location allocation and job scheduling. A two-layer genetic algorithm is designed to solve the model. The first layer is used to solve the location allocation, and the coding method is group coding; the second layer is used for job scheduling, and the coding mode is real number coding. When the population is initialized during the location allocation phase, the BFD algorithm is used to improve the convergence velocity of the algorithm. Taking the actual data of a tier-to-tier SBS/RS of an aviation food company as an example, the established model and design algorithm were verified, and the different batch intervals of each cargo space for storing different types of goods and outbound were analyzed. The optimization effects of the algorithm are compared, and the effects of considering the job scheduling and not considering the job scheduling on the location allocation are compared. The results show that based on the cargo allocation strategy of cargo mixed storage, the outbound efficiency can be improved by about $20 \%$. Considering job scheduling, the efficiency of warehousing is improved by about $5 \%$ compared with the optimization of warehouse allocation without job scheduling, where the efficiency of delivery is increased by about $6 \%$.
\end{abstract}

\section{Introduction}

Shuttle-based storage and retrieval system (SBS/RS) is a new fast access system in recent years. The system completes the task by cooperating with the elevator at the entrance and shuttle vehicle in the roadway. In order to improve the utilization ratio of shuttle vehicle, shuttle vehicle can access goods in different layers through elevator. Compared with the traditional automated storage and retrieval system (AS/RS), the cooperative operation of lifts and shuttle vehicles in SBS/RS greatly improves the operation efficiency of the system. In the logistics distribution of electronics, medicine, and aviation catering, the quantity of goods out of warehouse is relatively small and the variety is many. The warehousing operation is usually to select warehouses, and generally according to a certain batch out of warehouse, which is more suitable for the use of SBS/RS. Similar to AS/RS, location allocation is also a key issue affecting the operational efficiency of SBS/RS.

We have completed a project of AS/RS management system for an aviation catering enterprise. In the implementation of this project, we found that the storage efficiency of AS/RS is low. In this paper, SBS/RS is studied, which is applied to an aviation catering enterprise to replace the AS/RS. Picking is the main way of retrieval operation. In this paper, we study the mixed storage problem in SBS/RS and validate the optimization model 
and algorithm with the data of an aviation catering enterprise.

Storage location assignment problem has been extensively studied by scholars at home and abroad. Heskett [1] put forward the rule of cube-per-order index (COI). COI refers to the need of storage space divided by the number of times of goods out of storage per unit time. The larger the COI of goods is, the closer to the exit it should be stored. Carlo and Vis [2] studied a dynamic storage system, consisting of a conveyor, two nonpassing lifts that share a mast, multiple transfer shuttle vehicles, and a storage rack. This study is concerned with the scheduling problem of these two lifts. An integrated look-ahead strategy heuristic to simultaneously assign a set of predefined requests to the lifts and the order in which they will be handled taking into account delays caused by interference between the lifts is considered. A practical methodology to characterize the system to identify and resolve situations where the lifts would interfere with each other is presented. Lerher [3] proposed analytical travel time models for aisle changing shuttle vehicles, which are capable of travelling in the horizontal and cross-aisle directions. The expressions for the single- and dual-command travel times have been determined assuming uniform distributed storage locations and the probability theory. A simulation model has been applied for the performance analysis of the proposed analytical models. Ekren et al. [4] presented a tool that can calculate critical performance measures of different SBS/RS designs. By the provided tool, one can evaluate these systems' performance promptly by changing the input design parameters (e.g., discrete travel lengths, velocity of vehicles, etc.) of these systems. Borovinšek et al. [5] presented a multiobjective optimization solution procedure for the design of the SBS/RS. They considered three objective functions in the design concept, which are the minimization of average cycle time of transactions, amount of energy consumption, and total investment cost. During the optimization procedure, they considered seven design variables as number of aisles, number of tiers, number of columns, velocities of shuttle vehicle, acceleration/deceleration of shuttle vehicle, velocity of the elevators lifting tables, and acceleration/deceleration of the elevators lifting tables. They utilized the Non-Dominated Sorting Genetic Algorithm II (NSGA II) for facilitating the solution. Lerher et al. [6] presented an analysis of cycle times and throughput performances of SBS/RS. A parametric simulation model was developed for the purposes of this research. The model enables calculation of Single-Command (SC) and Dual-Command (DC) cycle times and throughput performances of SBS/RS. The model was tested on a case study, which is based on a real type of SBS/RS. Lerher et al. [7] presented a simulation-based performance evaluation of SBS/RS. Performance comparison of the studied SBS/RS was contrasted with alternative storage rack configurations, velocity profiles of the shuttle vehicle, and velocity profiles of the elevators' lifting tables in the system. The results showed that SBS/RSs are effective in reducing the mean cycle time and also showed large improvement by increasing throughput capacity. Lerher [8] presented an analytical travel time model for the computation of cycle times for double-deep SBS/RS. The proposed model considers the real operating characteristics of the elevators lifting table and the shuttle vehicle with the condition of rearranging blocking totes to the nearest free storage location during the retrieval process of the shuttle vehicle. Assuming uniform distributed storage locations and the probability theory, the expressions for the single- and dual-command cycle of the elevators lifting table and the shuttle vehicle have been determined. The proposed model enables the calculation of the expected cycle time for singleand dual-command cycles, from which the performance of the double-deep SBS/RS can be evaluated. Xie et al. [9] proposed a two-tier grouping optimization model to solve the storage location allocation problem with grouping constraints. Chen et al. [10] first proposed that the allocation of goods location should include two parts, the allocation of pick-up goods location and the allocation of inventory location, and studied the joint optimization of the allocation of goods location and job scheduling based on the strategy of shared goods location access. Ming et al. [11] proposed a method of goods location allocation in array automatic sorter based on the correlation of goods out of warehouse. The goods with strong warehouse correlation were allocated to distant channels to store. The efficiency of automatic sorter was improved by increasing the total virtual window time difference. Pan et al. [12] designed a heuristic genetic algorithm to solve the multiperson joint picking order picking allocation problem. Optimizing the storage location of goods is to balance the workload of each picking area, thereby improving the picking efficiency of the system. Pang and Chan [13] and others used data mining technology to extract and analyze the relationship between different products in customer orders. With the objective of minimizing the time of warehousing and picking operations, an optimization model of goods location allocation was established. Yang et al. [14] studied the integrated optimization of location allocation and job sequencing in multivehicle automatic access system under improved $2 n$ command cycle mode, established a quadratic integer programming model, and designed a tabu search algorithm to solve large-scale optimization problems. Yang et al. [15] studied the integrated optimization problem of multivehicle automated warehouse cargo location allocation and job scheduling, analyzed the instruction grouping, cargo location allocation and subtrip operation mode and sequence, considered the actual operation law of stacker, established an integrated optimization mathematical model with the minimum travel time as the objective function, and designed a double-layer genetic search algorithm to solve the model. The optimization effects of different order sizes and vehicle quantities are analyzed. Song et al. [16] studied the multiport access AS/RS and made a comprehensive analysis and research on the allocation of cargo location and order sequence. Gao [17], according to the relativity of traditional Chinese medicines in medical distribution orders, uses clustering method to store a variety of drug combinations in a storage space. The shuttle vehicle takes out a bin, which can select a variety of medicines, improves the efficiency of sorting, and reduces the number of uses of the storage space. 
Guo [18] puts forward two kinds of optimization problems for picking of single-load SBS/RS. Aiming at this problem, a model of picking and dispatching under the mode of 1-layer and $m$-layer and $n$-platform was established. The reasonable proportion of shuttle vehicle and elevator was determined by calculation and analysis. Zhang [19] analyzed the operation flow of the cross-layer shuttle vehicle system, established a semi-open-loop queuing network task scheduling model, and optimized the job queue of batch orders in a single time window.

Most of the existing research on location allocation only considers the situation that one location only stores one kind of goods, focusing on the integrated optimization of location allocation and job scheduling. Gao considered the mixed storage of goods, but did not consider the impact of job scheduling on the allocation of cargo space. Guo and Zhang studied the optimization of SBS/RS, but did not study the allocation of cargo space.

Therefore, in this paper, the optimization of storage location in tie-to-tie SBS/RS based on mixed cargo storage is studied.

\section{Problem Description}

SBS/RS is mainly composed of storage rack, shuttle vehicle, elevator, and so on, as shown in Figure 1. Depending on whether shuttle vehicles can perform cross-tier tasks with the help of a lift, two configurations can be defined: SBS/RS with tier-to-tier shuttle vehicles and that one with tier-captive shuttle vehicles. The tier-captive SBS/RS has a shuttle vehicle on each tier. The shuttle vehicle can only move on its own tier. The number of shuttle vehicles in tier-to-tier SBS/RS is less than the number of storage rack layers. The shuttle vehicle moves horizontally in the roadway and is responsible for the moving of the container between the cargo location and the caching platform. The elevator moves vertically on the side of the roadway. It is responsible for the moving of the container between the caching platform and the warehouse entry and exit platform, or for the replacement of the shuttle vehicle. Tier-to-tier SBS/RS is more flexible and stable than tier-captive SBS/RS. When the shuttle vehicle of one layer in the tier-captive SBS/RS fails, the cargo of that layer cannot be accessed and accessed, which affects the efficiency of the system. However, tier-to-tier SBS/RS can dispatch other shuttle vehicles into this layer to achieve cargo access. This paper mainly studies tier-to-tier SBS/ RS.

In the automated warehousing system, the allocation of storage space has an important impact on the utilization rate of warehouse and the efficiency of warehouse entry and exit. For inventory systems with small batches and many varieties, the storage capacity of warehouses can be improved by mixing storage of goods, reducing the number of goods used and increasing the storage capacity of warehouses.

For small batch and multivariety warehouse receipts, it is common to use multiple warehouse receipts as a batch for warehousing operations. Owing to the small batch size, it is generally a sorting operation. The sorting operation is a compound operation of one outgoing operation and one inputting operation. In order to avoid the elevator waiting for picking operation by pickers, ring conveyor belt can be designed as temporary storage area for picking operation. The size of the temporary storage area is designed according to the picking velocity and the running velocity of the elevator. The flow chart of the picking operation for tier-to-tier SBS/RS is shown in Figure 2.

To improve the operation efficiency of the automated warehousing system through the allocation of cargo space, the following aspects are mainly realized:

(1) To reduce the travel distance of stackers, shuttle vehicles, elevators, and other equipment through the allocation of cargo space. This is mainly achieved by storing goods with high frequencies into warehouses in short working hours.

(2) Through the allocation of cargo space, the operation times of stackers, shuttle vehicles, elevators, and other equipment are reduced. This is mainly through mixed storage of goods.

It can be realized by storing the goods that are often in the same warehouse receipt in the same warehouse location.

(3) Through the allocation of cargo space, the stackers in different lanes, shuttle vehicles in different layers, elevators, and other equipment can work in parallel as far as possible to reduce the idle waiting time of equipment. This is mainly achieved by storing the goods often in the same warehouse receipt in different roadways and different layers.

The problems studied in this paper can be described as follows: a tier-to-tier SBS/RS is composed of several roadways; each roadway has a single-load elevator, multiple replacement shuttle vehicles, and one shuttle vehicle replacement elevator; each cargo space only stores one container; each container can store a variety of goods; the way of warehousing is multivariety, small batch pickingout warehouse; in batches, multiple orders can be combined and sorted in batches according to the fixed time window set daily; there is a kind of goods stored in a warehouse system and mixed storage in a single warehouse; the correlation of goods out of warehouse is strong; based on the data of the warehouse receipt in a certain historical period, a plan of mixed storage and allocation of goods is designed to optimize the order of goods out of the same batch of warehouses so as to make this historical time. The shortest period of time is the period of out-of-warehouse operation.

\section{System Hypothesis and Model Establishment}

3.1. System Hypothesis. In order to facilitate research without losing generality, the following reasonable assumptions are made for the system: 


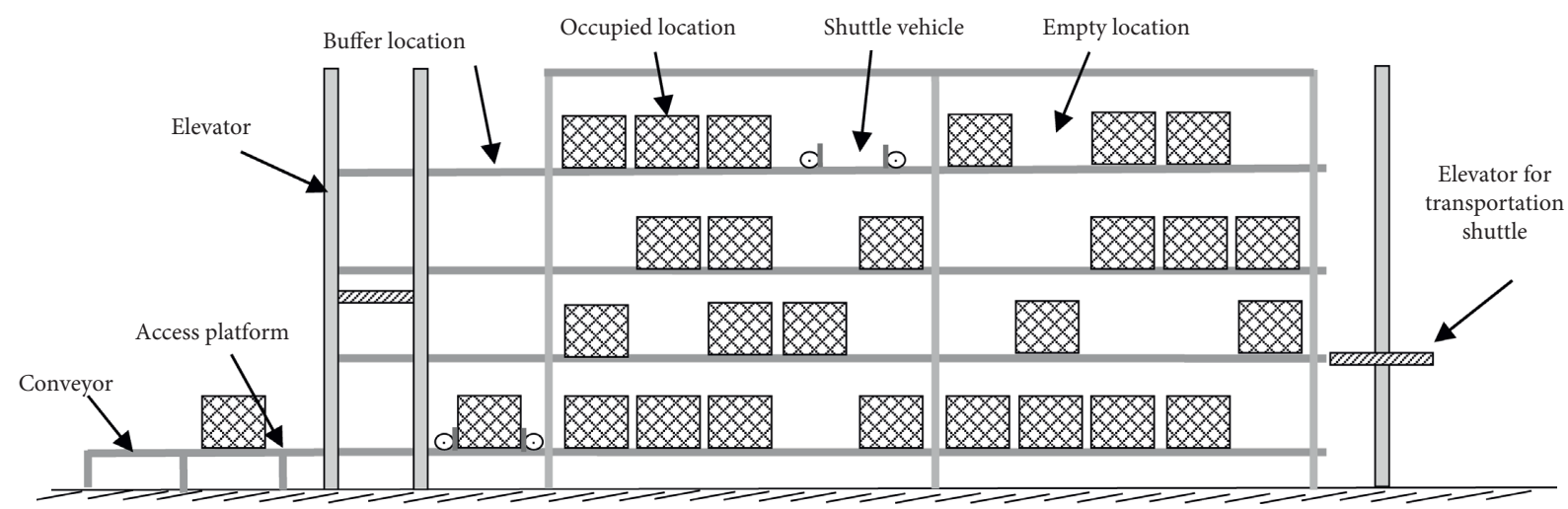

(a)

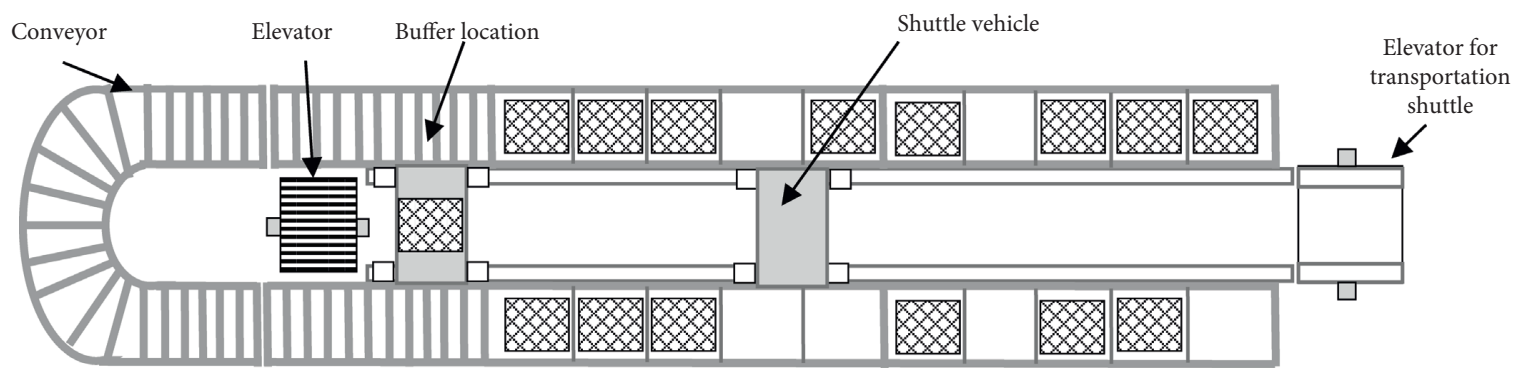

(b)

FIgURE 1: Schematic diagram of a tier-to-tier SBS/RS. (a) Main view diagram. (b) Overlooking sketch.

(1) Multiple cargoes can be stored in one storage location

(2) Each kind of cargoes is only stored in one location

(3) Each shuttle vehicle can work on any tier of the same aisle through the elevator that transports the shuttle vehicle

(4) The replenishment is timely, and there is no shortage of goods when they leave the warehouse

(5) The specifications of each location are the same

(6) The entrance and exit of the goods are on the first tier of the 0 th row of the roadway

(7) Pick in batches according to the fixed time window set daily, and at the same time select multiple outbound order

(8) The motion parameters of elevator and elevator for transport shuttle vehicle are the same

(9) The no-load and full-load motion parameters of elevator, shuttle vehicle, and elevator for transport shuttle vehicle are the same

(10) The acceleration of elevator and shuttle vehicle is constant and remains unchanged during acceleration and deceleration

(11) All types of outbound tasks are picking outbound

3.2. Model Establishment. The optimization objective of the system is to minimize the total operation time of all warehouse receipts in a certain period of history by optimizing the plan of mixed storage of goods and allocation of warehouse space, and the order of the same batch of goods out of warehouse.

The operation time of picking in tier-to-tier SBS/RS can be divided into the following parts:

(1) elevator operation time, including alignment time $t_{a}^{S}$, pick-up (or delivery) time $t_{b}^{S}$, travel time $t_{f_{s} f_{e}}^{S}$

(2) shuttle vehicle operation time, including alignment time $t_{a}^{C}$, pick-up (or delivery) time $t_{b}^{C}$, travel time $t_{l_{s} l_{e}}^{C}$, layer change time $t_{f_{s} f_{e}}^{C}$

(3) Picking conveyor belt conveyor time

(4) Picking time of pickers

$t_{a}^{C}, t_{b}^{C}, t_{a}^{S}$ and $t_{b}^{S}$ are the design parameters of the equipment, which can be obtained according to the system parameters. In the course of elevator and shuttle vehicle movement, it includes start-up, uniform motion, and braking. Known from the literature [15], the changes of acceleration and velocity with time during the movement of the elevator's lifting table and shuttle vehicle are shown in Figure 3. In Figure 1(a), the travel distance of the elevator's lifting table or shuttle vehicle is relatively long, and its movement process is as follows: start-acceleration- uniform velocity-deceleration- stop. In Figure 1(b), the travel distance of the elevator or shuttle vehicle is relatively short, and its movement process is as follows: start-acceleration-decelerationstop.

In both cases, the calculation equation for the travel time of the elevator's lifting table or shuttle vehicle is shown in equation (1). 


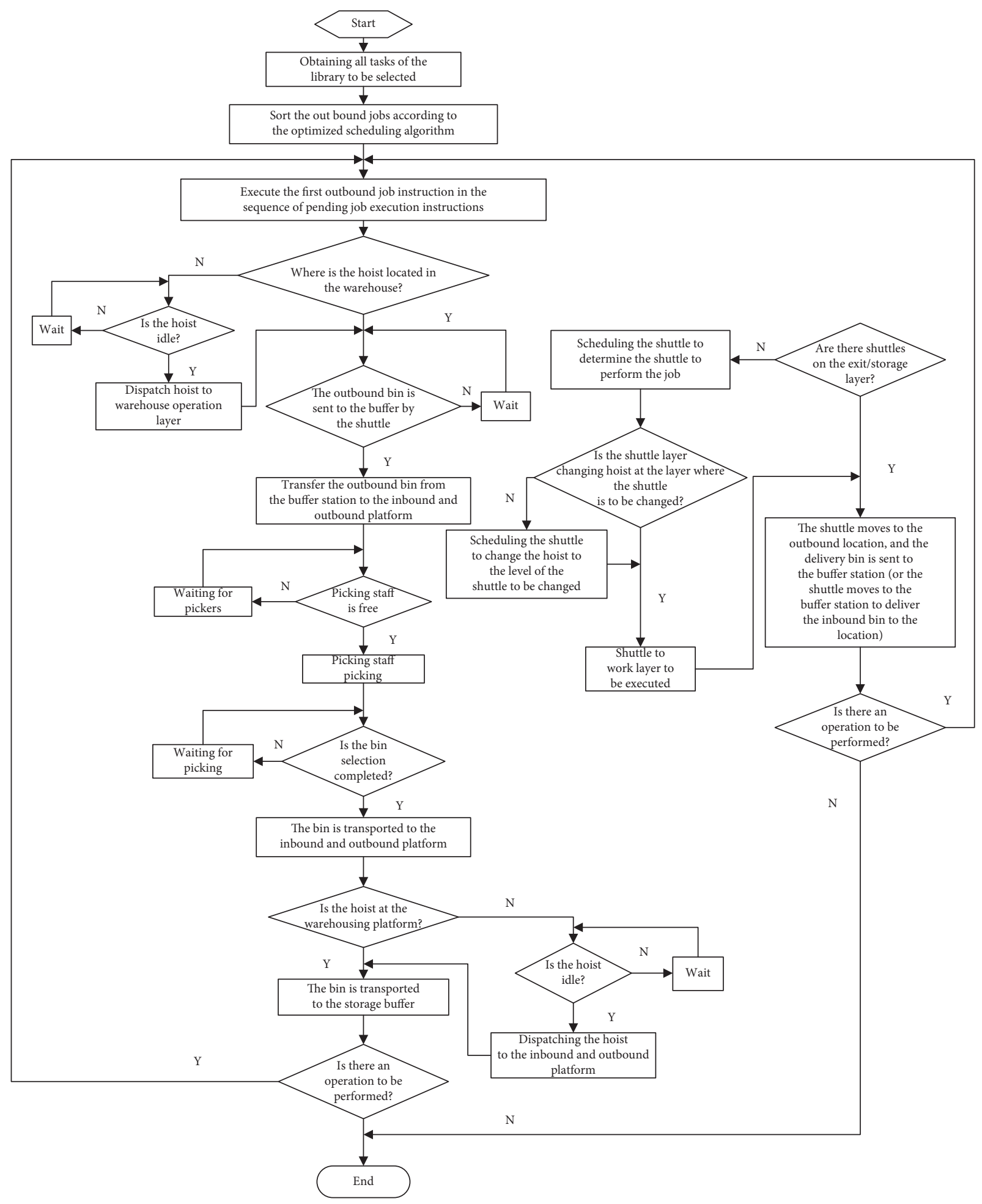

Figure 2: Picking process of tier-to-tier SBS/RS.

$$
t= \begin{cases}2 v_{\max } \cdot a^{-1}+\left(s-v_{\max }^{2} \cdot a^{-1}\right) \cdot v_{\max }^{-1}=v_{\max } \cdot a^{-1}+s \cdot v_{\max }^{-1}, & s \geq \frac{v_{\max }^{2}}{a}, \\ 2 \sqrt{s \cdot a^{-1}}, & s<v_{\max }^{2} \cdot a^{-1}\end{cases}
$$




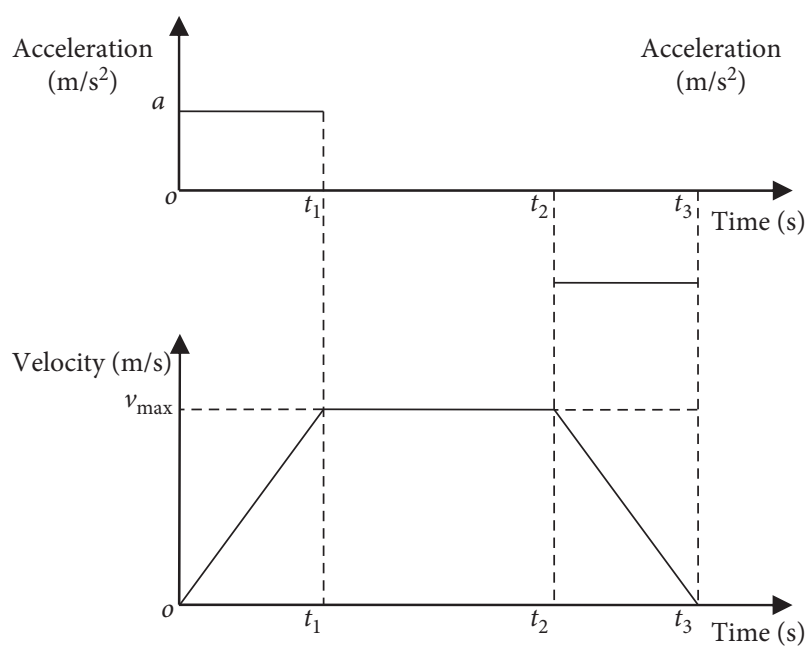

(a)

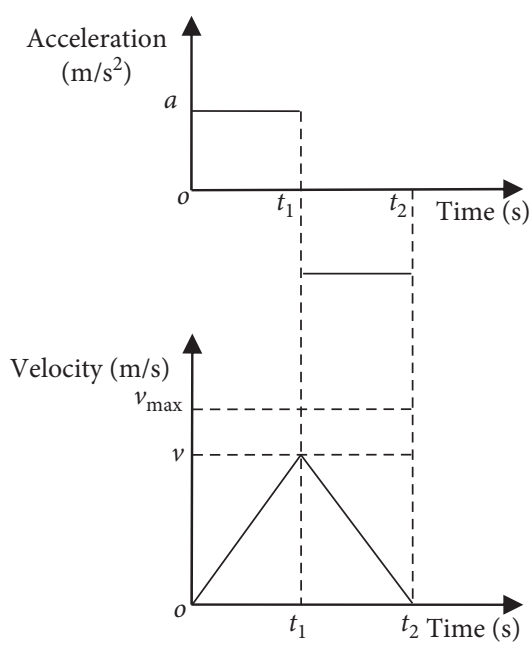

(b)

Figure 3: Time dependency of the elevator and the shuttle vehicle.

Referring to equation (1), the calculation of equipment travel time is carried out. The equations are as follows:

$$
\begin{aligned}
t_{f_{s} f_{e}}^{S} & = \begin{cases}v_{s} \cdot a_{s}^{-1}+\left|f_{e}-f_{s}\right| \cdot H \cdot v_{s}^{-1}, & \left|f_{e}-f_{s}\right| \geq v_{s}^{2} \cdot a_{s}^{-1}, \\
2 \sqrt{\left|f_{e}-f_{s}\right| \cdot H \cdot a_{s}^{-1}}, & \left|f_{e}-f_{s}\right|<v_{s}^{2} \cdot a_{s}^{-1},\end{cases} \\
t_{l_{s} l_{e}}^{C} & = \begin{cases}v_{c} \cdot a_{c}^{-1}+\left|l_{e}-l_{s}\right| \cdot L \cdot v_{c}^{-1}, & \left|l_{e}-l_{s}\right| \geq v_{c}^{2} \cdot a_{c}^{-1}, \\
2 \sqrt{\left|l_{e}-l_{s}\right| \cdot L \cdot a_{c}^{-1}}, & \left|l_{e}-l_{s}\right|<v_{c}^{2} \cdot a_{c}^{-1} .\end{cases}
\end{aligned}
$$

Passing equation (3), calculating the Layer Change Time of shuttle vehicle Truck $t_{f_{s} f_{e}}^{C}$ :

$$
t_{f_{s} f_{e}}^{C}=\max \left(t_{l_{s(0)} l_{h}}^{C}+t_{a}^{C}+t_{a}^{S}+2 t_{b}^{S}+t_{f_{s} f_{e}}^{S}, t_{f_{s(2)} f_{s}}^{S}+2 t_{a}^{S}+2 t_{b}^{S}+t_{f_{s} f_{e}}^{S}\right) .
$$

The picking time of the pickers is related to the picking velocity and quantity of the pickers. It is not convenient to calculate the picking time. Conveyor belt conveying time is affected by picking time, and it is not convenient to calculate.
In order to calculate the operation time and make reasonable assumptions, it is assumed that, in a batch of picking warehouse operations, the picking personnel work fast enough, the length of conveyor belt is reasonable, and there is no situation where the elevator waits for the picking personnel to carry out the picking operation. Therefore, when calculating the operation time of the picking library, only the operation time of the elevator and shuttle vehicle is calculated. Assuming that the elevator completes a pick-up operation and the picker can complete a pick-up operation of the bin, the order of execution of the pick-up operation in lane $N^{O_{d k q}}$ of lane $q$ of the batch $k\left(k \in B_{d}\right)$ on day $d$ is as follows:

The first outgoing operation $\longrightarrow$ the second outgoing operation $\longrightarrow$ the first inputting operation $\longrightarrow$ the third outgoing operation $\longrightarrow \ldots \longrightarrow$. The $N^{O_{d k q}}-1$ warehousing operation and the $N^{O_{d k q}}$ warehousing operation. A total of $N^{O_{d k q}}-2$ pairs of in-out composite operations, 2 out-ofwarehouse operations and 2 in-warehouse separate operations.

The working hours of the equipment that performs a single outgoing operation are shown in

$$
T_{O}=\max \left(\begin{array}{c}
t_{f_{s(0)} f_{e(0)}}^{S}+t_{f_{e(0)} f_{o}}^{S}+2 t_{a}^{S}+2 t_{b}^{S}, \\
t_{l_{s(0)} l_{e(O)}}^{C}+t_{l_{e(O)} l_{o}}^{C}+2 t_{a}^{C}+2 t_{b}^{C}+t_{f_{s(1)} f_{e(0)}}^{C}+t_{f_{e(0)} f_{o}}^{S}+t_{a}^{S}+t_{b}^{S}
\end{array}\right),
$$

The working hours of the equipment that performs a single warehousing operation are as follows:

$$
T_{I}=\max \left(\begin{array}{c}
t_{f_{s(0)} f_{o}}^{S}+t_{f_{o} f_{e(I)}}^{S}+2 t_{a}^{S}+2 t_{b}^{S}+t_{l_{o} l_{e(I)}}^{C}+t_{a}^{C}+t_{b}^{C}, \\
t_{l_{s(0)} l_{o}}^{C}+t_{l_{o} l_{e(I)}}^{C}+2 t_{a}^{C}+2 t_{b}^{C}+t_{f_{s(1)} f_{e(I)}}^{C}
\end{array}\right) .
$$


The working hours of performing one storage and one outward composite operation equipment are as follows:
(1) The inbound and outbound locations are at the same level.

$$
T_{I O}=\max \left(\begin{array}{c}
t_{f_{o} f_{e(I)}}^{S}+2 t_{a}^{S}+4 t_{b}^{S}+t_{f_{e(I)} f_{o}}^{S} \\
t_{l_{s(0)} l_{e(O)}}^{C}+t_{l_{e(0)} l_{o}}^{C}+2 t_{a}^{C}+2 t_{b}^{C}+t_{f_{s(1)} f_{e(O)}}^{C}+t_{a}^{S}+2 t_{b}^{S}+t_{f_{e(I)} f_{o}}^{S} \\
t_{l_{s(0)} l_{e(O)}}^{C}+t_{l_{e(O)} l_{o}}^{C}+4 t_{a}^{C}+4 t_{b}^{C}+t_{f_{s(1)} f_{e(O)}}^{C}+t_{l_{o} l_{e(I)}}^{C}
\end{array}\right) .
$$

(2) The inbound and outbound positions are not at the same level.

$$
T_{I O}=\max \left(\begin{array}{c}
t_{f_{o} f_{e(I)}}^{S}+2 t_{a}^{S}+4 t_{b}^{S}+t_{f_{e(I)} f_{e(O)}}^{S}+t_{f_{e(0)} f_{o}}^{S}, \\
t_{l_{s(0)} l_{o}}^{C}+t_{l_{o} l_{e(I)}}^{C}+2 t_{a}^{C}+2 t_{b}^{C}+t_{f_{s(1)} f_{e(I)}}^{C} \\
t_{l_{s(0)} l_{e(O)}}^{C}+t_{l_{e(O)} l_{o}}^{C}+2 t_{a}^{C}+2 t_{b}^{C}+t_{f_{s(1)} f_{e(O)}}^{C}+t_{a}^{S}+2 t_{b}^{S}+t_{f_{e(O)} f_{o}}^{S}
\end{array}\right) .
$$

The total operation time $T_{d k q}$ of the $N^{O_{d k q}}$ picking libraries is shown in

$$
T_{d k q}= \begin{cases}T_{O(1)}+T_{O(2)}+\sum_{o=1}^{N_{d k q}} T_{I O(o)}+T_{I\left(N^{O_{d k q}}\right)}+T_{I\left(N^{O_{d k q}}\right)}, & N^{O_{d k q}}>2, \\ T_{O(1)}+T_{O(2)}+T_{I(1)}+T_{I(2)}+T_{w}, & N^{O_{d k q}}=2, \\ T_{O(1)}+T_{I(1)}+T_{w}, & N^{O_{d k q}}=2 .\end{cases}
$$

When $T_{d k q}=1$ or $T_{d k q}=2$, there is an elevator waiting for picking time $T_{w}$. Under batch picking, generally $T_{d k q}>2$. Therefore, when calculating the total picking time, $T_{w}$ is ignored. $T_{d k}$ :

On day $d$, the total time of $k\left(k \in B_{d}\right)$ outgoing batches is

$$
T_{d k}=\max \left\{T_{d k q}(q \in R)\right\} .
$$

The total discharge time $T$ of a certain period in history is

$$
T=\sum_{d \in D} \sum_{k \in B_{d}} T_{d k}
$$

Under the strategy of mixed storage and batch sorting of goods, the relationship between the location of goods and the time of goods leaving the warehouse is established through the following processes:

(1) Obtain the cargo set $\operatorname{ORDER}_{d k}$ in the $k\left(k \in B_{d}\right)$-th warehouse release order on day $d(d \in D)$, and merge the same cargo in different warehouse release orders.
(2) Search the location of the goods and generate the set of operation instructions $O_{d k}$ of the $k\left(k \in B_{d}\right)$-out batch on day $d(d \in D)$ and $J$ of the $q(q \in R)$-lane operation instructions $O_{d k q}$ of the $k\left(k \in B_{d}\right)$-out batch on day $d(d \in D)$ according to the location of the goods. The elements in the set of operation instructions are represented by the outbound location, and the decision variable $x_{i j}$ is used to determine whether the retrieval operation instruction exists in the location $j$.

$$
\begin{aligned}
O_{d k q}= & \left\{\operatorname{LOC}_{1} \bigcup_{i \in \mathrm{ORDER}_{d k}} x_{i 1}, \mathrm{LOC}_{2} \bigcup_{i \in \mathrm{ORDER}_{d k}} x_{i 2}, \ldots,\right. \\
& \left.\operatorname{LOC}_{j\left(q, 2, \mathrm{~N}^{\mathrm{LNE}}, N^{F}\right)} \bigcup_{i \in \mathrm{ORDER}_{d k}} x_{i j\left(q, 2, \mathrm{~N}^{\mathrm{LNE}}, N^{F}\right)}\right\} .
\end{aligned}
$$

(3) The elements of instruction set $O_{d k q}$ are sorted by decision variable $x_{o o}^{\prime}$. Decision variable $x_{o o}^{\prime}$ can be represented by matrix $X$. 


$$
X=\left|\begin{array}{cccc}
0 & 1 & \cdots & 0 \\
0 & 0 & \cdots & 1 \\
\vdots & \vdots & \vdots & \vdots \\
1 & 0 & \cdots & 0
\end{array}\right|
$$

One line in $X$ is all 0 , indicating that the instruction is at the end; the other is all 0 , indicating that the instruction is at the front, and it is not at the end of any instruction.

The ordered instruction set $O_{d k q}^{\prime}$ is

$$
O_{d k q}^{\prime}=O_{d k q} \cdot X
$$

The optimization process can be divided into two stages: the first stage is to optimize the allocation of mixed storage space for goods, and the second stage is to optimize the order of picking warehouse operations.

The optimization objectives are as follows:

$$
\begin{aligned}
& \min T=\sum_{d \in D} \sum_{k \in B_{d}} \max \left\{T_{d k q}(q \in R)\right\}, \\
& \text { s.t. } \quad \sum_{j \in \mathrm{LOC}} x_{i j}=1, \quad \forall i \in G \\
& \sum_{i \in G} x_{i d k} \geq 1, \quad \forall k \in B_{d}, \\
& \sum_{i \in G} x_{i j} \leq N^{G M a x}, \quad \forall j \in \mathrm{LOC}, \\
& \sum_{o \in O_{d k q}} x_{o o}^{\prime} \leq 1, \quad \forall o^{\prime} \in O_{d k q}, \\
& \sum_{o, \in O_{d k q}} x_{o o}^{\prime} \leq 1, \quad \forall o \in O_{d k q}, \\
& x_{o o}^{\prime}=0, \quad o=o^{\prime}, \\
& \sum_{i=1}^{n} N_{i} V_{i} x_{i j} \leq V_{\max }, \quad \forall j \in \text { LOC, } \\
& \sum_{i=1}^{n} N_{i} W_{i} x_{i j} \leq W_{\max }, \quad \forall j \in \text { LOC. }
\end{aligned}
$$

Equation (15) denotes that only one kind of goods is stored in one place, equation (16) denotes that one lot contains one or more kinds of goods, equation (17) denotes that the maximum number of goods that can be stored in one place is $N^{G M a x}$, equations (18) and (19) denote that the order of execution of operation instructions is uniquely determined, and equation (20) denotes that the order of execution of the same instruction can not be followed by equation (21). The volume of all goods stored in Place $j(j \in$ LOC) shall not be greater than the maximum storage volume of Place B. Equation (22) means that the weight of all goods stored in Place $j(j \in$ LOC) shall not be greater than the maximum storage weight of Place $B$.
In an automated warehousing system, the maximum quantity (called "maximum storage quota") that a certain kind of goods can be stored in a single warehouse is generally set to meet the storage volume and weight limitation of the warehouse. For example, the maximum storage limit of a certain type of disposable lunch boxes in a warehouse is 2000; that is, a storage space can store up to 2000 of this type of lunch boxes, which can not only ensure that the total volume does not exceed the maximum storage volume of a storage space, but also ensure that the total weight does not exceed the maximum storage weight of a storage space. Therefore, constraints (21) and (22) can be merged into

$$
\sum_{i \in G} \frac{N_{i} x_{i j}}{N_{\max (i)}} \leq 1 .
$$

Equation (23) denotes that the sum of the quantities of goods stored in Place $j(j \in \mathrm{LOC})$ and the ratio of the maximum storage limit for each of them are less than or equal to 1 .

\section{Algorithm Design}

The optimization model of cargo location allocation established in this paper includes two stages: the first stage is to allocate cargo storage location; the second stage is to optimize the execution sequence of cargo location operations according to the historical outbound list data and the way of batch outbound. According to the established objective function, the paper evaluates the advantages and disadvantages of location allocation through the completion time of outgoing operations in a certain period of history and explains the influence of operation execution sequence on location allocation.

Based on the allocation of goods in mixed storage, it can be understood as a special packing problem. In automated warehousing system, in order to facilitate access and storage, when goods are mixed, different kinds of goods can not be stacked. Whether a variety of goods can be mixed into the bin depends on whether multiple cargo bottoms can be assembled into the bottom of the bin. This problem can be understood as a special two-dimensional packing problem. Two-dimensional packing problem is a typical combinatorial optimization problem, which belongs to NP-hard problem. Many scholars have studied the solution of twodimensional packing problem. Thomas and Chaudhari [20] proposed a hyperheuristic algorithm based on genetic algorithm to solve two-dimensional packing problem. Polyakovskiy and M'Hallah [21] proposed a two-dimensional packing problem with mixed feasibility constraints guiding search to the due date. Lodi et al. [22] proposed a partial enumeration algorithm for two-dimensional packing problem with truncation constraints. The solution of twodimensional packing problem is very complicated. The main objective of this paper is to improve the efficiency of the multilayer shuttle vehicle system. Although there are constraints on the quantity of goods stored, the quantity of goods stored is dynamic, and precise constraints have no significant impact on the solution of the problem. Therefore, 
in order to simplify the solution of the problem, this paper transforms the two-dimensional packing problem into onedimensional packing problem, without considering the effect of cargo shape and size on packing. Zhang et al. [23] designed a hybrid genetic algorithm by using group representation to code and improved the solution of packing problem. Although genetic algorithm has strong global search ability and can obtain better solution, the main problem is that it converges to the global optimal solution slowly and has poor timeliness. Aiming at the problems existing in solving the packing problem by genetic algorithm, Zhang et al. [24] designed an improved genetic algorithm. By adding the descending optimal adaptive algorithm, the initialization population was generated, the optimal individual preservation strategy was selected, and the evaluation scale of fitness was transformed, which improved the solving velocity of genetic algorithm and the probability of finding the optimal solution.

Regarding the job scheduling problem of automated warehousing system, Krishnaiah Chetty et al. [26] used genetic algorithm to solve the dynamic job scheduling problem. The results show that genetic algorithm is better than NN (Nearest Neighbor). Gharehgozli et al. [26] optimize the order of access jobs, effectively reducing the total travel time of S/R machine in dual-inventory automatic access system. Yang et al [27]. analyzed the actual dispatching path of elevator and shuttle vehicle in the system, established the corresponding mathematical model considering the acceleration and deceleration characteristics of elevator and shuttle vehicle in the process of motion, and designed the hybrid plant propagation algorithm to solve the problem.

Genetic algorithm is a highly parallel, stochastic and adaptive optimization algorithm based on the survival of the fittest. It has global convergence under certain conditions. It has been widely used to solve optimization problems such as packing problem, job shop scheduling problem, and TSP problem. In this paper, a two-level genetic algorithm is designed based on the idea of hierarchical optimization. By using the feedback relationship between different optimization levels, the obtained solution is improved in time to obtain the optimal solution of the integrated optimization problem.

The basic flow of the algorithm is as follows: firstly, the optimization is started from the mixed storage of goods and the allocation of storage space, and the inventory goods are mixed in the empty storage space according to the constraints. Then, according to the storage location of the goods, the operation instructions of batch out of the historical warehouse receipt are generated. Then the job instructions of each batch are optimized, and the shuttle vehicle that executes the retrieval operation instructions is allocated to determine the execution order of the job instructions and calculate the job time of each batch so as to obtain the total job time of a certain period in history. The job instruction scheduling layer continuously iterates and optimizes to get the optimal job time and then returns to the allocation layer to redistribute the location. Through several iterations, the optimal allocation result is finally obtained. The flow chart of the algorithm is shown in Figure 4 (see the next page).

4.1. Chromosome Coding Design. When genetic algorithm is used to solve packing problem, its coding can be expressed in three ways: (1) box-based representation, (2) item-based representation, and (3) group-based representation [17]. Falkenauer [18] proposes a group-based representation of packing problem, which consists of two parts: the first part encodes the box, and the second part indicates which items are stored in which box. The chromosome structure based on population coding is that the location of the gene represents the storage location, the value of the gene represents all the items stored in the storage location, and the length of the chromosome is the number of storage sites. This paper chooses group coding method. In order to express conveniently, the simplified numbering in natural number form is adopted for both location and goods. The chromosome of genetic algorithm in the stage of allocation of goods is shown in Figure 5.

This coding representation enables genes to represent both goods and cargo locations. The principle is that, in the packing problem, genetic operators only operate on the population part of chromosomes, and the goods part only indicates which cargo the population consists of.

In the job scheduling stage, this paper uses real-number coding based on the outgoing location.

\subsection{Initial Population Generation}

4.2.1. Location Allocation Stage. Among the heuristic algorithms to solve the packing problem, the first-Fit Decreasing (FFD) algorithm and the Best-Fit Decreasing (BFD) algorithm are two better off-line algorithms. In order to enhance the search ability of genetic algorithm and find the closest optimal solution to packing problem, FFD or BFD algorithm can be used to generate dominant individuals when initializing population.

When initializing the population, this paper designs the following heuristic algorithm based on FFD and BFD algorithm.

Step 1: Establish ITEM for goods to be stored and sort them according to COI rules.

Step 2: Establish the LOCATION of the allocated freight space set, and rank it from near to far from the export.

Step 3: Establish CITEM of allocated goods, select $i$ of the lowest COI in ITEM, allocate $j$ of the nearest location in LOCATION, store the goods $i$ of $j$ in CITEM, and delete $i$ and $j$ of allocated goods from ITEM and LOCATION, respectively.

Step 4: Choose goods $i^{\prime}$ whose similarity coefficient is the largest in set ITEM and allocated location $j$, and the quantity of goods in storage is less than the surplus capacity of allocated location. If $i^{\prime}$ exists, store goods $i^{\prime}$ in location $j$ and delete it from set ITEM. If $i^{\prime}$ does not exist and ITEM is not empty, then go to step 3 and 


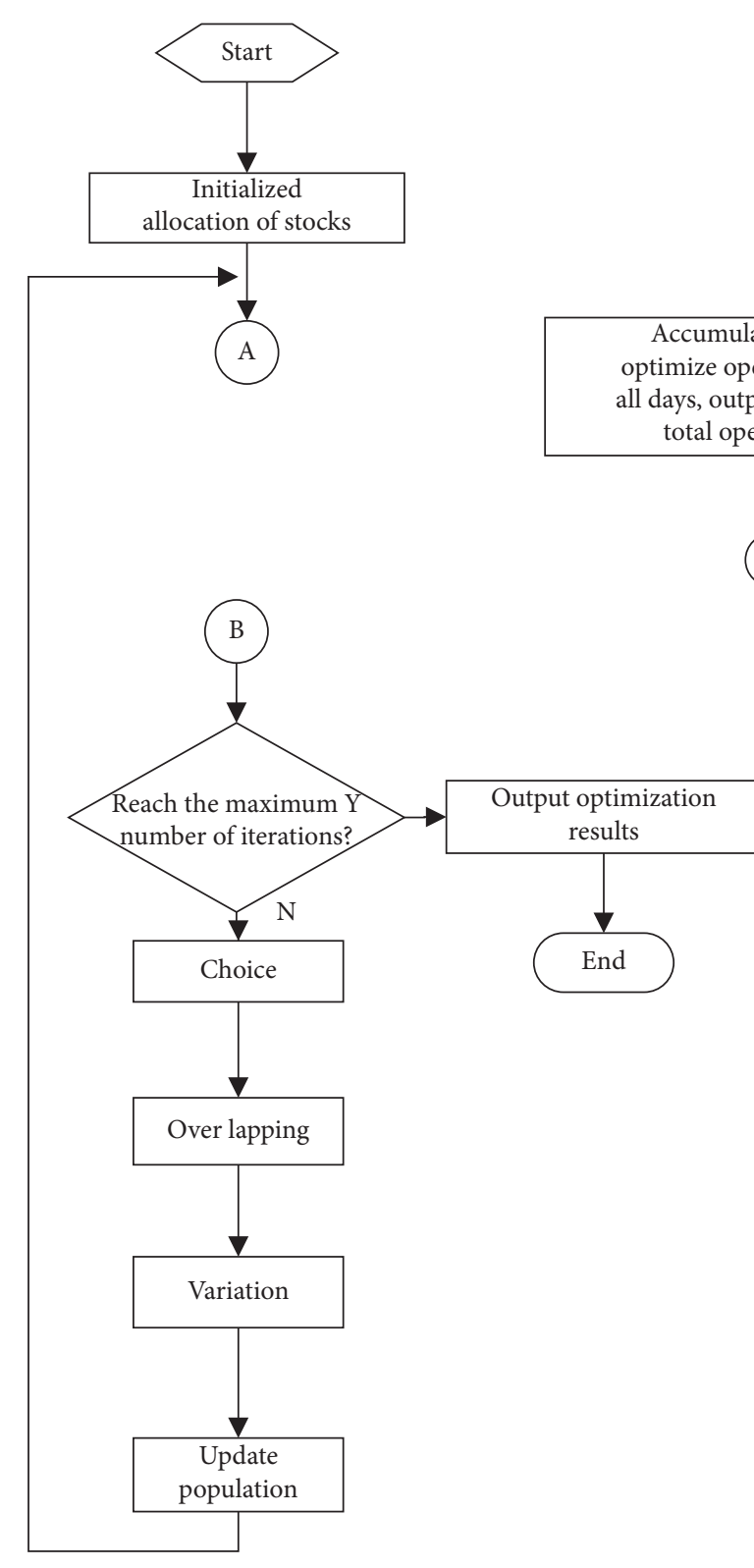

According to the individual allocation of the population, the batch operation instruction set is generated for a certain period of history.
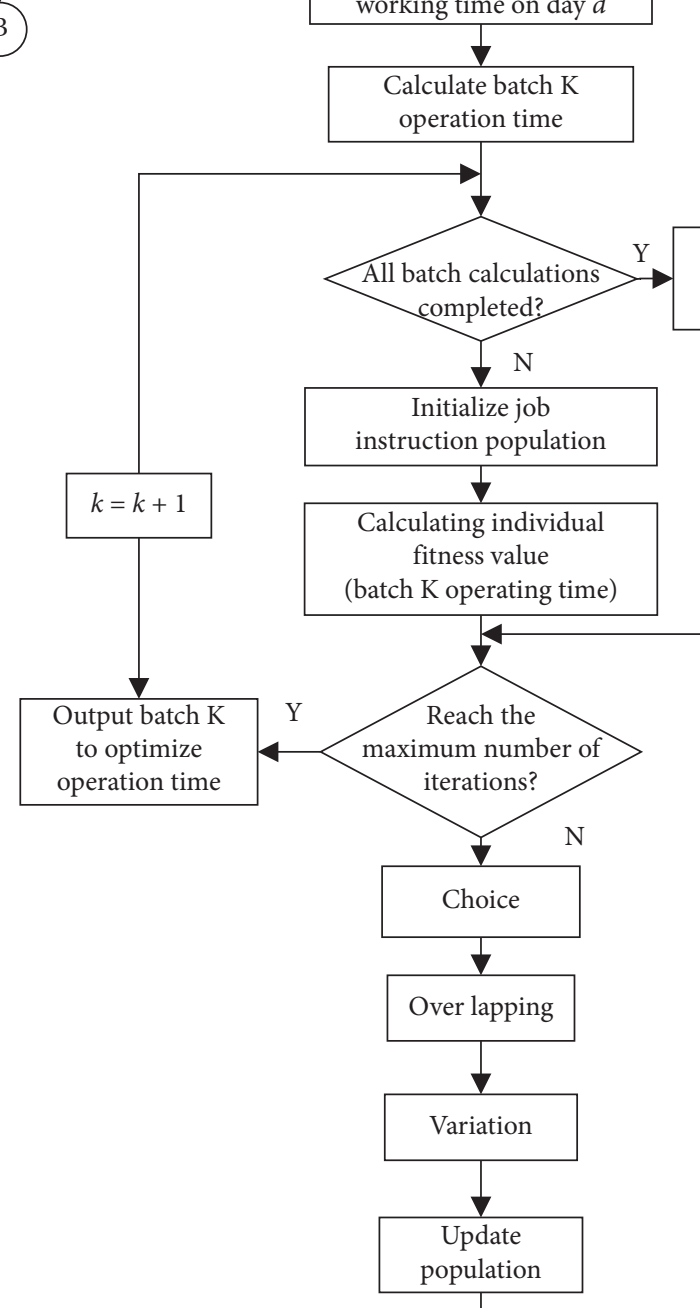

FIGURE 4: Flow chart of bilayer genetic algorithm.

\begin{tabular}{|c|c|c|c|c|}
\hline 1 & 2 & 3 & 4 & Simplified location numbering \\
\hline $1,3,7$ & 2,8 & 4,10 & $5,6,9$ & Simplified goods numbering \\
\hline
\end{tabular}

Figure 5: Chromosome coding method in the location allocation stage. 
select the next location $j+1$ to store the goods. If ITEM is empty, LOCATION is the first individual in the initial population.

Step 5: Randomly select two locations, exchange the goods stored, and create a new individual.

For the selection of population size, more is better in theory, but considering the calculation cost, satisfactory results can be obtained. Generally, the population size ranges from 10 to 100 .

4.2.2. Job Scheduling Phase. In the sequencing stage of job instructions, because the chromosome coding is real number coding, the randomly generated chromosomes are all feasible solutions. Therefore, the initial population is generated randomly.

4.3. Fitness Calculation and Population Selection. Roulette selection, ranking and proportion transformation, competitive selection, and so on are commonly used selection methods of genetic algorithm. The most well-known and commonly used selection method is roulette selection. In roulette selection, the maximum of fitness function is usually obtained, so the objective function is transformed into equation (24):

$$
f=\frac{1}{\min T} .
$$

This paper adopts elite retention strategy when using roulette selection method. The idea of this strategy is to copy the best individuals (called elite individuals) that have emerged so far in the evolutionary process directly to the next generation without pair crossover. The advantage of this strategy is that the best individuals will not be lost and destroyed by selection, crossover, and mutation operations. Elite retention strategy plays an important role in improving the global convergence ability of standard genetic algorithm.

4.4. Crossover Operator. The crossover operation in genetic algorithm is the core step of population evolution. Two paternal chromosomes exchange gene fragments in different ways to produce offspring chromosomes. The commonly used methods of crossover are single parent inheritance, double parent-son crossover, and multiparent crossover. In order to avoid the infeasibility of the crossover operation, the parent-child crossover method is adopted and the subindividuals are generated according to the corresponding constraints.

4.4.1. Location Allocation Stage. The intersection process in the allocation stage is shown in Figure 6.

The cross-over process is described as follows.

Step 1: Choose two parents and randomly choose one crossing position.
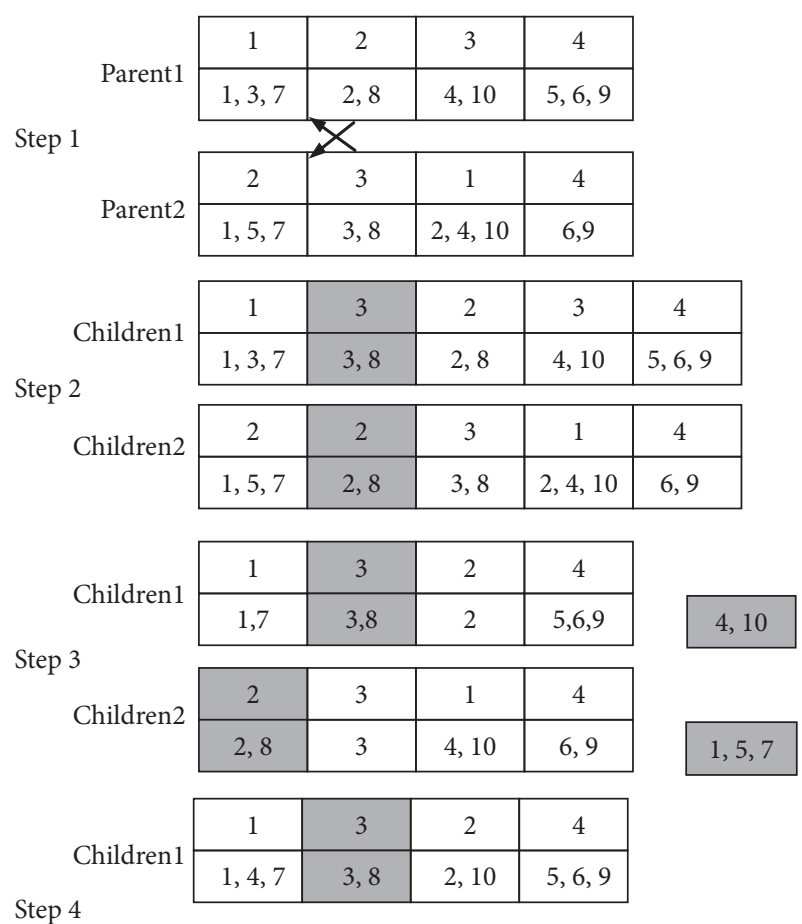

\begin{tabular}{|c|c|c|c|c|c|}
\hline \multirow{2}{*}{ Children2 } & 2 & 3 & 1 & 4 & 5 \\
\cline { 2 - 6 } & 2,8 & $1,3,5$ & 4,10 & 6,9 & 7 \\
\hline
\end{tabular}

FIGURE 6: Intersection process of cargo location allocation phase.

Step 2: Select the intersecting parts of two parents, insert them into the intersecting position of another parent, and produce two offspring.

Step 3: Remove all duplicate goods from the resulting offspring;

Step 4: Remove duplicate cargo location (if there is no duplicate cargo location, remove the cargo location with the least type or occupying the least storage space). If the remaining cargo can be put into other cargo locations, according to BFD heuristic algorithm, put it into another cargo location; if it can not be put into other cargo locations, then put it into a new cargo location.

4.4.2. Job Instruction Sequencing Phase. The crossover process of job instruction sequencing phase is described as follows:

Step 1: Choose two parents and randomly choose one crossing position.

Step 2: Select the intersecting parts of two parents, insert them into the intersecting position of another parent, and produce two offspring.

Step 3: Remove all duplicate locations in the resulting offspring.

4.5. Mutation Operator. The mutation operator affects the local search ability and population diversity of the algorithm. 
In the stage of allocation, this paper adopts group coding. The mutation operation rules are as follows: random selection of a mutation location, removal of the location of the goods, and according to the BFD heuristic method, the goods removed from the location are put into other locations; if they can not be put into other locations, then put them into new locations.

4.5.1. Location Allocation Stage. The variation process in the allocation stage is shown in Figure 7:

4.5.2. Job Scheduling Phase. In order to avoid generating invalid solutions with the same nodes, the mutation operation rules are designed as follows: randomly select a certain number of chromosomes according to the mutation probability, perform single parent mutation operation on the selected chromosomes, randomly generate two mutation positions, and then put the two gene positions into each other. Exchange generates new subindividuals, and the new individuals must correspond to feasible solutions.

\section{Example Analysis}

5.1. Instance Data. In order to verify the correctness of the optimization model and the validity of the optimization algorithm for the allocation of cargo space in the interlayer shuttle vehicle storage system based on hybrid storage, the data of the interlayer shuttle vehicle storage system of an aeronautical food company is selected as an example. During the peak period, the airline food company has more than 90,000 meals a day. There are nearly 700 kinds of ingredients and raw materials for meals. The warehousing system is frequently operated in and out of the warehouse. The tier-totier SBS/RS consists of three lanes, six rows of shelves, three cargo elevators, three elevators for transport shuttle vehicle, and 12 shuttle vehicles. Each shuttle vehicle is responsible for fixing two tiers, and the storage rack scale is 8 tiers and 45 rows, with the same cargo space specifications. The basic parameters of the equipment are shown in Table 1.

By analyzing the data of the cross-layer shuttle vehicle warehousing system from August to October 2018, 80 kinds of goods with high frequency and less quantity are selected as the test objects of cargo location allocation. The number of warehousing orders including 80 kinds of goods is 1436 , and the number of warehousing operation instructions is 5232 . The warehousing information of some goods is shown in Table 2.

5.2. Calculation and Analysis. The program is programmed with MATLAB R2014 and tested under WIN64 bit operating system, 4GB memory, and Intel Core (TM) i5-6500 $(3.2 \mathrm{GHz})$ environment. The parameters of genetic algorithm are as follows: population size 100, crossover probability 0.8 , mutation probability 0.15 , and iteration times 200; the parameters of genetic algorithm in job scheduling stage are as follows: population size 30 , crossover probability 0.8 , mutation probability 0.15 , and iteration times 50 . The
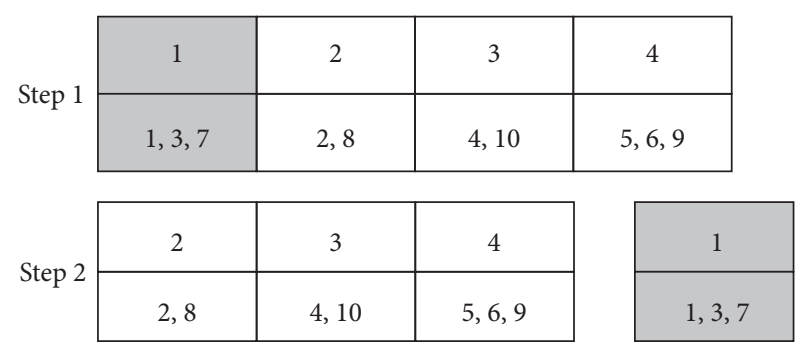

\begin{tabular}{|c|c|c|c|}
\hline \multirow{2}{*}{ Step 3} & 3 & 4 & 1 \\
\cline { 2 - 5 } & 2 & 3 & 4,8 \\
\hline
\end{tabular}

FIGURE 7: Variation process in cargo location allocation stage.

TABLE 1: Basic equipment parameters.

\begin{tabular}{lcc}
\hline Parameter name & Symbol & Parameter values \\
\hline Cargo height & $H$ & $0.6 \mathrm{~m}$ \\
Cargo location length & $L$ & $0.8 \mathrm{~m}$ \\
Elevator acceleration & $a_{s}$ & $3 \mathrm{~m} / \mathrm{s}^{2}$ \\
Maximum velocity of elevator & $v_{s}$ & $4 \mathrm{~m} / \mathrm{s}$ \\
Elevator alignment time & $t_{a}^{S}$ & $0.5 \mathrm{~s}$ \\
Elevator pick-up (or delivery) time & $t_{b}^{S}$ & $1.5 \mathrm{~s}$ \\
Shuttle vehicle acceleration & $a_{c}$ & $2 \mathrm{~m} / \mathrm{s}^{2}$ \\
Maximum velocity of shuttle vehicle & $v_{c}$ & $4 \mathrm{~m} / \mathrm{s}$ \\
Shuttle vehicle alignment time & $t_{a}^{C}$ & $0.5 \mathrm{~s}$ \\
Shuttle vehicle pick-up (or delivery) & $t_{b}^{C}$ & $3 \mathrm{~s}$ \\
time & &
\end{tabular}

model is simulated and calculated. Each case scheme runs 20 times, and the average value of 20 times is obtained from the experimental results.

\subsubsection{Testing of the Maximum Number of Different Varieties} Stored in Each Shipping Space. When the maximum number of goods stored in each location is different, the maximum number of goods that can be stored in each location is set to $1-8$, respectively, and the time interval of batch operation of daily warehouse discharge list is set to 1 hour. The test results are shown in Table 3.

It can be known from Table 3 that the retrieval operation time for storing only one type of goods at each location is the longest. With the increase of the number of types of goods stored in each location, the retrieval operation time is getting shorter and shorter, but the shortening range of the retrieval operation time is smaller and smaller. The outbound time of 8 types of goods stored in each location is only $0.09 \%$ less than the outbound time of 7 types of goods stored in each location.

With the increase of the types of goods stored in each storage location, the complexity of goods storage operation will increase, and the efficiency of goods inbound will decrease. In practical application, the outbound efficiency of the goods and the inbound efficiency of the goods should be considered comprehensively to determine the optimal number of goods types stored in each storage location. 
TABLE 2: Warehousing information of goods.

\begin{tabular}{lcccc}
\hline $\begin{array}{l}\text { Simplified numbering of } \\
\text { goods }\end{array}$ & Product name & Unit & $\begin{array}{c}\text { Single input } \\
\text { quantity }\end{array}$ & Single capacity maximum capacity \\
\hline 1 & West lake longjing green tea (10 g) & Bag & 3000 & 10000 \\
2 & Qi Hong special grade $(50 \mathrm{~g})$ & Bag & 500 & 1350 \\
3 & Bagged Jasmine (10 g) & Bag & 2400 & 6300 \\
4 & First class head nuts & Bag & 4800 & 12000 \\
5 & 750 mL great wall dry white wine & Bottle & 240 & 360 \\
6 & Blue canned yanjing beer (our company) & Bottle & 600 & 1512 \\
7 & French Mediterranean knight dry white & Bottle & 120 & 396 \\
8 & wine & & 600 \\
9 & Soma-wipe toilet paper (eastern airlines) & Bag & 200 & \\
10 & First-class towel & Strip & 1800 & 4800 \\
$\ldots$ & Fig payment (luxury first class) washing & One & 120 & $\ldots$ \\
\hline
\end{tabular}

TABLE 3: The simulation results of the maximum number of different varieties stored per location.

\begin{tabular}{lcccc}
\hline $\begin{array}{l}\text { Maximum number of items per } \\
\text { storage }\end{array}$ & $\begin{array}{c}\text { Initial solution } \\
(\mathrm{s})\end{array}$ & $\begin{array}{c}\text { Optimum solution } \\
(\mathrm{s})\end{array}$ & $\begin{array}{c}\text { Algorithm calculation } \\
\text { time }(\mathrm{s})\end{array}$ & $\begin{array}{c}\text { Optimal solution improvement rate } \\
(\%)\end{array}$ \\
\hline 1 & 625770 & 539178 & 386 & - \\
2 & 540618 & 441508 & 405 & 18.11 \\
3 & 481098 & 408474 & 431 & 7.48 \\
4 & 442852 & 387220 & 446 & 5.20 \\
5 & 435614 & 375621 & 462 & 3.00 \\
6 & 431027 & 371258 & 470 & 1.16 \\
7 & 429945 & 369144 & 475 & 0.57 \\
8 & 429122 & 368827 & 481 & 0.09 \\
\hline
\end{tabular}

* Optimal solution improvement rate (OSIS) is that the reduction rate of the optimal solution in this row is relative to the optimal solution in the previous row. Take the second row of data for example, OSIS $=(539178-441508) \div 539178=18.11 \%$.

In the example of this paper, if a maximum of 4-5 types of goods are stored in one location, the SBS/RS outbound efficiency is higher.

5.2.2. Testing of Different Shuttle Vehicle Numbers. In order to test the optimization effect of location allocation based on mixed storage of goods in the case of SBS/RS system with different number of shuttle vehicles, it is assumed that the number of shuttle vehicles configured in the system is $1-8$, and the maximum number of goods stored in each location is 4 . The test results are shown in Table 4 .

It can be seen from Table 4 that the optimization model established in this paper and the optimization algorithm designed in this paper can achieve better optimization results under the condition that different numbers of shuttle vehicle are configured. With the increase in the number of shuttle vehicle buses, the retrieval operation time is decreasing, but the decreasing range is becoming smaller. As the number of shuttle vehicle buses increases, the load rate of the elevator increases gradually, and the average load rate of shuttle vehicle buses decreases gradually. When the shuttle vehicle bus number is 4 , the load rate of the elevator is $79.2 \%$, and the average load rate of the shuttle vehicle is $69.83 \%$, which is relatively good configuration.
5.2.3. Testing the Impact of Job Scheduling on Location Allocation. In order to analyze the effect of job scheduling on the allocation of cargo location, according to the maximum number of goods stored in each cargo location, simulation is carried out separately without considering job scheduling and job scheduling, and comparative analysis is made. When job scheduling is considered, the batch job interval is set to 1 hour. The simulation data are shown in Table 5.

As shown in Table 5, it is more efficient to consider job scheduling than not to consider job scheduling. With the increase of the number of varieties stored in each storage space, the improvement effect decreases. This is because when the types of goods stored in each location increase, the outbound times required for the same batch of outbound orders will decrease. As the number of jobs decreases, the effectiveness of job scheduling decreases. Therefore, the impact of job scheduling needs to be considered when conducting SBS/RS storage allocation.

5.2.4. Convergence Analysis of Algorithms. The results of several runs of the algorithm show that the program can converge in a finite number of iterations and obtain the optimal results. The time interval of batch operation for each warehouse order is 1 hour. Each warehouse can store up to 1, 
TABLE 4: Simulation results for different numbers of shuttle vehicles.

\begin{tabular}{lcccc}
\hline $\begin{array}{l}\text { Number of shuttle } \\
\text { vehicles }\end{array}$ & $\begin{array}{c}\text { Optimum solution } \\
(\mathrm{s})\end{array}$ & $\begin{array}{c}\text { Elevator load rate } \\
(\%)\end{array}$ & $\begin{array}{c}\text { Shuttle vehicle average load } \\
\text { rate }(\%)\end{array}$ & $\begin{array}{c}\text { Optimal solution improvement rate } \\
(\%)\end{array}$ \\
\hline 1 & 1151743 & 25.08 & 100 & - \\
2 & 698714 & 46.07 & 87.89 & 39.33 \\
3 & 472597 & 61.83 & 81.56 & 32.36 \\
4 & 386952 & 79.2 & 69.83 & 18.12 \\
5 & 328175 & 87.34 & 49.93 & 15.19 \\
6 & 289289 & 92.9 & 40.67 & 11.85 \\
7 & 258173 & 96.41 & 41.26 & 10.76 \\
8 & 235426 & 98.32 & 33.5 & 8.81 \\
\hline
\end{tabular}

TABLE 5: Comparison of the effect of job scheduling on outbound job time.

\begin{tabular}{lccc}
\hline Maximum number of items per storage & Regardless of job scheduling (s) & Consider job scheduling (s) & Improvement rate (\%) \\
\hline 1 & 586324 & 539178 & 8.04 \\
2 & 471522 & 441508 & 6.37 \\
3 & 430190 & 408474 & 5.05 \\
4 & 403877 & 387200 & 4.13 \\
5 & 388251 & 375621 & 3.25 \\
6 & 381769 & 371258 & 2.75 \\
7 & 377218 & 369144 & 2.14 \\
8 & 375916 & 368827 & 1.89 \\
\hline
\end{tabular}

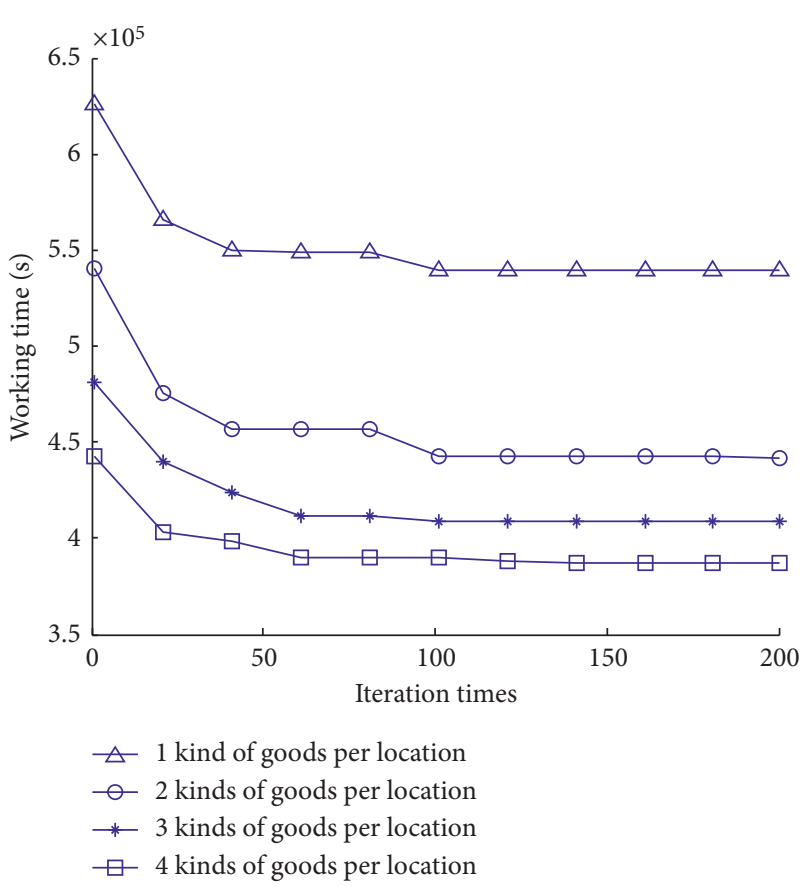

FIgURE 8: Convergence curve of the algorithms.

2, 3, and 4 kinds of goods, respectively. The convergence curve of the algorithm is shown in Figure 8.

From Figure 8, it can be seen that the algorithm can converge in 100 operations with an interval of 1 hour and a maximum of 1-4 kinds of goods stored in each location, reflecting the good convergence of the algorithm.

\section{Conclusion}

Considering the mixed storage of goods, this paper studies the optimal allocation of cargo space in the tier-to-tier SBS/
RS. This paper analyzes the solving process of operation time in batch picking warehouse, which is carried out by the combination of outgoing and inputting operations. An optimal allocation model is established to minimize the total time of all out-of-warehouse orders in a certain historical period. The model considers the effect of job scheduling on the allocation of goods and is divided into two stages: the allocation of goods and job scheduling. Two-level genetic algorithm is designed to solve the model. Each layer of genetic algorithm designs coding mode, crossover operator, and mutation operator, respectively.

This paper takes the actual data of a tier-to-tier SBS/RS of an airline food company as an example, verifies the established model and designed algorithm from different aspects, and draws the following conclusions:

(1) The retrieval operation time for storing only one type of goods at each location is the longest. With the increase of the number of types of goods stored in each location, the retrieval operation time is getting shorter and shorter, but the shortening range of the retrieval operation time is smaller and smaller. With the increase of the types of goods stored in each storage location, the complexity of goods storage operations will increase, and the efficiency of goods inbound will decrease. In practical application, the outbound efficiency of the goods and the inbound efficiency of the goods should be considered comprehensively to determine the optimal number of goods types stored in each storage location. In the example of this paper, if a maximum of 4-5 types of goods are stored in one location, the SBS/RS outbound efficiency is higher.

(2) The optimization model established in this paper and the optimization algorithm designed in this paper can achieve better optimization results under the 
condition that different numbers of shuttle vehicle are configured. With the increase in the number of shuttle vehicle buses, the retrieval operation time is decreasing, but the decreasing range is becoming smaller. As the number of shuttle vehicle buses increases, the load rate of the elevator increases gradually, and the average load rate of shuttle vehicle buses decreases gradually. When the shuttle vehicle bus number is 4 , the load rate of the elevator is $79.2 \%$, and the average load rate of the shuttle vehicle is $69.83 \%$, which is relatively good configuration.

(3) It is more efficient to consider job scheduling than not to consider job scheduling. With the increase of the number of varieties stored in each storage space, the improvement effect decreases. This is because when the types of goods stored in each location increase, the outbound times required for the same batch of outbound orders will decrease. As the number of jobs decreases, the effectiveness of job scheduling decreases. Therefore, the impact of job scheduling needs to be considered when conducting SBS/RS storage allocation.

For the optimization of SBS/RS location allocation based on cargo mixed storage, the following content needs further study:

(1) Optimization of location allocation for the same kind of goods stored in multiple locations

(2) Optimization of storage location allocation for mixed storage of goods in shelves with different layer-to-column ratios

(3) The influence of the quantity, weight, and volume of the goods on the mixed storage of the goods

\section{Abbreviations}

AS/RS: Automated storage and retrieval systems

GA: Genetic algorithm

I/O: Input and output location

SBS/RS: Shuttle-based storage and retrieval systems

\section{Symbols}

$G: \quad$ Is the inventory goods category set, index $i$, the number of types of goods is $N^{G}$

LOC: Location set, index $j$, total number of goods is $N^{\mathrm{LOC}}$

Q: $\quad$ Rack roadway number set, indexed as $q$

F: $\quad$ Storage rack level set, indexed as $f, N^{F}$ for storage rack tiers

LINE: is the collection of storage rack columns indexed as $l, N^{\mathrm{LINE}}$ is the number of storage racks

$R: \quad$ is the collection of storage rack rows indexed as $r, R \in\{1,2\}$

$N^{G M a x}$ : is the maximum number of types of goods that can be stored at each location

$\operatorname{LOC}_{j_{(q, r, l, f)}}$ : indicates that the roadway where $\mathrm{LOC}_{j}$ is located is $q(q \in Q)$, platoon as $r(r \in R)$, listed as $l(l \in \mathrm{LINE})$, layer for $f(f \in F)$

$D$ : $\quad$ Set of working days in a certain period of history, indexed to $d$

$B_{d}: \quad$ Batch collection sorted in batches on day $d(d \in D)$, indexed to $k$

ORD: The collection of warehouse release orders in a certain period of history

$\mathrm{ORD}_{d}: \quad$ The collection of warehouse discharge orders on day $d(d \in D)$

$\mathrm{ORD}_{d k}: \quad$ On day $d(d \in D)$, batch $k\left(k \in B_{d}\right)$ is a collection of warehouse receipts

$O_{d k}: \quad$ The set of instructions for batch $k\left(k \in B_{d}\right)$ out of warehouse on day $d(d \in D)$

$O_{d k q}: \quad$ The set of operation instructions for roadway $q(q \in R)$ in batch $k\left(k \in B_{d}\right)$ on day $d(d \in D)$, indexed by $o$ and $o l, N^{\mathrm{O}_{d k q}}$ is the number of instructions

$N_{i}: \quad$ The quantity of goods $i(i \in G)$ in storage at a single time

$V_{i}: \quad$ Unit volume of goods $i(i \in G)$

$W_{i}: \quad$ Unit weight of goods $i(i \in G)$

$V_{\max }: \quad$ Maximum storage volume for a single cargo location

$W_{\max }: \quad$ Maximum storage weight of single location

$N_{\max (i)}: \quad$ The maximum quantity of goods $i(i \in G)$ stored in a single location

$H$ : $\quad$ Cargo height

L: $\quad$ Cargo location length

$a_{s}: \quad$ Elevator acceleration

$v_{s}: \quad$ Maximum velocity of elevator

$a_{c}: \quad$ Shuttle vehicle acceleration

$v_{c}$ : $\quad$ Maximum velocity of shuttle vehicle

$t_{a}^{C}: \quad$ Shuttle vehicle alignment time

$t_{b}^{C}$ :

$t_{a}^{S}:$

$t_{b}^{S}$

$t_{l_{s} l_{e}}^{C}$ :

$t_{f_{s} f_{e}}^{S}$ :

$t_{f_{s} f_{e}}^{C}:$

$l_{s(0)}:$

$l_{o}$ :

$l_{h}:$

$l_{e(I)}$ :

$l_{e(O)}:$

$f_{s}:$

$f_{e}:$

$f_{s(0)}:$

$f_{s(1)}:$

$f_{s(2)}:$
Shuttle vehicle pick-up (or delivery) time

Elevator alignment time

Elevator pick-up (or delivery) time

The travel time of shuttle vehicle from column $l_{s}\left(l_{s} \in \mathrm{LINE}\right)$ to $l_{e}\left(l_{e} \in \mathrm{LINE}\right)$

Elevator travel time from layer $f_{s}\left(f_{s} \in F\right)$ to Layer $f_{e}\left(f_{e} \in F\right)$

The travel time of shuttle vehicle from layer $f_{s}\left(f_{s} \in F\right)$ to Layer $f_{e}\left(f_{e} \in F\right)$

The shuttle vehicle standby position is in the row Buffer area is defined as the rank 0

The shuttle vehicle replacement layer is in the row, that is, the last row of the storage rack

The rank of the warehouse cargo location

The rank where the warehouse cargo is located The shuttle vehicle standby position is in the layer The shuttle vehicle target layer The level where the elevator standby is located Shuttle vehicle stand-by level The shuttle vehicle replacement elevator standby position is in the layer 


\begin{tabular}{|c|c|}
\hline$f_{o}:$ & $\begin{array}{l}\text { The level where the platform is located, that is, } \\
\text { the first level }\end{array}$ \\
\hline$f_{e(I)}:$ & The level where the warehouse cargo is located \\
\hline & The level where the warehouse cargo is located \\
\hline & evator waiting for picking time \\
\hline 1 & xecute a single warehousing operation time \\
\hline$\sigma^{\circ}$ & xecution of a single retrieval operation time \\
\hline & $\begin{array}{l}\text { Execute } 1 \text { input and } 1 \text { output compound job } \\
\text { time }\end{array}$ \\
\hline$\Gamma:$ & $\begin{array}{l}\text { Total operating time of all warehouse receipts at } \\
\text { a certain period of time in history }\end{array}$ \\
\hline$T_{d}$ & Total outgoing time of date $d(d \in D)$ \\
\hline$T_{d k}:$ & $\begin{array}{l}\text { The total operation time of batch } k\left(k \in B_{d}\right) \text { on } \\
\text { day } d(d \in D)\end{array}$ \\
\hline$T_{d k q}:$ & $\begin{array}{l}\text { The total working time of roadway } q(q \in R) \text { in } \\
\text { the batch } k\left(k \in B_{d}\right) \text { on day } d(d \in D)\end{array}$ \\
\hline${ }^{l}(q, r, l, f)$ & $\begin{array}{l}\text { Decision variables, } x_{i j_{(q, r, l, f)}}=1 \text {, it means that } \\
\text { the goods } i(i \in G) \text { are stored in the No. } \\
j_{(q, r, l, f)}\left(j \in \text { LOC) slot, } x_{i j_{(q, r, l f)}}=0 \text { means that }\right. \\
\text { the goods } i \text { are not stored in the no. } j \text { slot. }\end{array}$ \\
\hline$x_{o o}:$ & $\begin{array}{l}\text { Decision variables, } x_{o o}^{\prime}=1 \text {, it denotes that } \\
\text { instruction } o^{\prime}\left(o^{\prime} \in O_{d k q}\right) \text { is executed after } \\
\text { picking library instruction } o\left(o \in O_{d k q}\right) \text {, and } \\
x_{o o}^{\prime}=0 \text { denotes that instruction is executed after } \\
\text { picking library instruction } o \text { is not } o \text {. }\end{array}$ \\
\hline
\end{tabular}

\section{Data Availability}

The data used in this study are taken from the work field database, which can be provided if necessary.

\section{Conflicts of Interest}

The authors declare that they have no conflicts of interest.

\section{References}

[1] J. Heskett, "Cube-per-order-a key to warehouse stock location," Transportation and Distribution Management, vol. 2, pp. 27-31, 1963.

[2] H. J. Carlo and I. F. A. Vis, "Sequencing dynamic storage systems with multiple lifts and shuttles," International Journal of Production Economics, vol. 140, no. 2, pp. 844-853, 2012.

[3] T. Lerher, "Aisle changing shuttle carriers in autonomous vehicle storage and retrieval systems," International Journal of Production Research, vol. 56, no. 11, pp. 3859-3879, 2018.

[4] B. Y. Ekren, A. Akpunar, Z. Sari, and T. Lerher, "A tool for time, variance and energy related performance estimations in a shuttle-based storage and retrieval system," Applied Mathematical Modelling, vol. 63, pp. 109-127, 2018.

[5] M. Borovinšek, B. Y. Ekren, A. Burinskienè, and T. Lerher, "Multi-objective optimisation model of shuttle-based storage and retrieval system," Transport, vol. 32, no. 2, pp. 120-137, 2017.

[6] T. Lerher, M. Borovinsek, M. Ficko, and I. Palcic, "Parametric Study of throughput performance in SBS/RS based on simulation," International Journal of Simulation Modelling, vol. 16, no. 1, pp. 96-107, 2017.

[7] T. Lerher, Y. B. Ekren, Z. Sari, and B. Rosi, "Simulation analysis of shuttle based storage and retrieval systems,"
International Journal of Simulation Modelling, vol. 14, no. 1, pp. 48-59, 2015.

[8] T. Lerher, "Travel time model for double-deep shuttle-based storage and retrieval systems," International Journal of Production Research, vol. 54, no. 9, pp. 2519-2540, 2016.

[9] J. Xie, Y. Mei, A. T. Ernst, X. Li, and A. Song, "A bi-level optimization model for grouping constrained storage location assignment problems," IEEE Transactions on Cybernetics, vol. 48, no. 1, pp. 385-398, 2018.

[10] L. Chen, A. Langevin, and D. Riopel, "The storage location assignment and interleaving problem in an automated storage/ retrieval system with shared storage," International Journal of Production Research, vol. 48, no. 4, pp. 991-1011, 2010.

[11] M. Li, Y. Wu, J. Zhang et al., "Slotting optimization of array automated picking device based on item correlation," Computer Integrated Manufacturing Systems, vol. 21, no. 7, pp. 1896-1905, 2015.

[12] J. C.-H. Pan, P.-H. Shih, M.-H. Wu, and J.-H. Lin, “A storage assignment heuristic method based on genetic algorithm for a pick-and-pass warehousing system," Computers \& Industrial Engineering, vol. 81, no. 3, pp. 1-13, 2015.

[13] K.-W. Pang and H.-L. Chan, "Data mining-based algorithm for storage location assignment in a randomised warehouse," International Journal of Production Research, vol. 55, no. 14, pp. 4035-4052, 2017.

[14] P. Yang, Y. Peng, B. Ye, and L. Miao, "Integrated optimization of location assignment and sequencing in multi-shuttle automated storage and retrieval systems under modified $2 n$ command cycle pattern," Engineering Optimization, vol. 49, no. 9, pp. 1604-1620, 2017.

[15] W. Yang, T. Yue, J. Liu et al., "Routing optimization for compound operations of the double-loading multi-tier shuttle vehicle warehousing system," Computer Integrated Manufacturing Systems, vol. 24, no. 12, pp. 3178-3188, 2018.

[16] Y. B. Song, Z. Y. Jiang, and B. Z. Sun, "Integrated optimization of operations in multiple-I/O points automated storage and retrieval system," Journal of Central South University (Science and Technology), vol. 47, no. 06, pp. 1930-1939, 2016.

[17] Y. Gao, A Study of Packing Strategy of Mini-Load Automated Storage and Picking System in Medical Logistics Distribution Center, Shandong University, Jinan, China, 2016.

[18] H. L. Guo, Optimization Research of Scheduling on Automated Warehouse Based on Multishuttle, Beijing Wuzi University, Beijing, China, 2013.

[19] N. Zhang, Modeling and Optimization of Tier-to-Tier Shuttlebased Storage and Retrieval System, Shandong University, Jinan, China, 2018.

[20] J. Thomas and N. S. Chaudhari, "Design of efficient packing system using genetic algorithm based on hyper heuristic approach," Advances in Engineering Software, vol. 73, pp. 45-52, 2014.

[21] S. Polyakovskiy and R. M'Hallah, "A hybrid feasibility constraints-guided search to the two-dimensional bin packing problem with due dates," European Journal of Operational Research, vol. 266, no. 10, pp. 819-839, 2017.

[22] A. Lodi, M. Monaci, and E. Pietrobuoni, "Partial enumeration algorithms for two-dimensional bin packing problem with guillotine constraints," Discrete Applied Mathematics, vol. 217, no. 1, pp. 40-47, 2017.

[23] D. Zhang, G. Liu, J. Wang et al., "Genetic algorithm based on group coding for bin-packing problem," Computer Engineering and Design, vol. 29, no. 12, pp. 3154-3156, 2008.

[24] Y. J. Zhang, Y. Liu, and S. J. Xie, "An improved genetic algorithm for bin-packing problem," Control Engineering of China, vol. 23, no. 03, pp. 327-331, 2016. 
[25] O. V. Krishnaiah Chetty and M. Sarveswar Reddy, "Genetic algorithms for studies on AS/RS integrated with machines," International Journal of Advanced Manufacturing Technology, vol. 22, no. 1, pp. 932-940, 2003.

[26] A. H. Gharehgozli, Y. Yu, X. Zhang, and R. d. Koster, "Polynomial time algorithms to minimize total travel time in a two-depot automated storage/retrieval system," Transportation Science, vol. 51, no. 1, pp. 19-33, 2017.

[27] W. Yang, T. Yue, J. Liu et al., "Routing optimization for compound operations of the double-loading multi-tier shuttle warehousing system," Computer Integrated Manufacturing Systems, vol. 24, no. 12, pp. 3178-3188, 2018. 